\title{
Relationship between photonic band structure and emission characteristics of a polymer distributed feedback laser
}

\author{
G. A. Turnbull, ${ }^{1}$ P. Andrew, ${ }^{2}$ M. J. Jory, ${ }^{2}$ W. L. Barnes,${ }^{2, *}$ and I. D. W. Samuel ${ }^{1, *}$ \\ ${ }^{1}$ Ultrafast Photonics Collaboration, School of Physics and Astronomy, University of St Andrews, \\ St Andrews, Fife KY16 9SS, United Kingdom \\ ${ }^{2}$ Thin Film Photonics Group, School of Physics, University of Exeter, Stocker Road, Exeter EX4 4QL, United Kingdom
}

(Received 10 April 2001; published 11 September 2001)

\begin{abstract}
We present an experimental study of the emission characteristics and photonic band structure of a distributed feedback polymer laser, based on the material poly[2-methoxy-5-(2'-ethylhexyloxy)-1,4-phenylene vinylene]. We use measurements of the photonic band dispersion to explain how the substrate microstructure modifies both spontaneous and stimulated emission. The lasing structure exhibits a one-dimensional photonic band gap around $610 \mathrm{~nm}$, with lasing occurring at one of the two associated band edges. The band edge (frequency) selection mechanism is found to be a difference in the level of output coupling of the modes associated with the two band edges. This is a feature of the second-order distributed feedback mechanism we have employed and is clearly evident in the measured photonic band structure.
\end{abstract}

DOI: 10.1103/PhysRevB.64.125122

PACS number(s): 42.55.-f, 42.70.Jk, 42.70.Qs

\section{INTRODUCTION}

In recent years, semiconducting conjugated polymers have received considerable interest as novel laser gain media. ${ }^{1-14}$ They exhibit optical gain over broad spectral ranges through the visible, and so are well suited to use in tuneable lasers ${ }^{4}$ or broadband amplifiers. ${ }^{5,6}$ Unlike other organic chromophores they exhibit little concentration quenching, and may readily be used undiluted in the solid state. Such a configuration may be compatible with electrical excitation $^{2,3}$ using the maturing technology of polymer lightemitting diodes (LEDs). While electrically pumped lasing has recently been demonstrated in organic crystals, ${ }^{15}$ the prospect of polymer diode lasers fabricated by simple processing from solution is very appealing.

Such simple processing to produce high quality thin films has allowed demonstrations of polymer lasers in various resonator geometries. ${ }^{2,3}$ These include microcavities, ${ }^{7}$ microrings, ${ }^{8}$ distributed feedback, ${ }^{4,9,10}$ and photonic band-gap ${ }^{11,12}$ resonators, and even random lasing structures. ${ }^{13,14}$ Usually the conjugated polymer is coated onto a previously shaped or textured substrate to produce the desired form. In the above configurations the emitting layer is formed into a wavelength-scale photonic structure which may in turn modify the polymer's emission properties. Through active control of the photonic modes it should be possible to control lasing properties such as threshold and output coupling. It is therefore important to understand how the emission of the polymer is modified by the photonic environment.

In this paper we use direct measurements of the photonic band structure to explain the spectral features of the emission from a distributed feedback polymer laser. By doing so, we obtain insight into the factors that control lasing in these systems. Specifically, we present a study of the operating characteristics of a distributed feedback laser based on the conjugated polymer poly(2-methoxy-5-(2'-ethylhexyloxy)1,4-phenylene vinylene) (MEH-PPV). We describe its emis- sion properties when operating both below and above lasing threshold, and relate these properties to the photonic mode structure of the device. Such a comparison is found to be helpful in understanding the mechanisms that control emission from the polymer. It is thus a useful technique in the general study of photonic microstructures and, in particular, in the drive towards low threshold polymeric lasers suitable for electrical excitation.

\section{LASER FABRICATION AND EXPERIMENTAL PROCEDURE}

The structure of the polymer distributed feedback (DFB) laser studied is shown schematically in Fig. 1(a). It is comprised of a corrugated silica substrate covered by a thin film of the conjugated polymer MEH-PPV. The silica-polymer-air structure formed an asymmetric slab waveguide that supported only the fundamental transverse electric mode for the wavelengths of interest.

The substrate corrugations were initially defined holographically in a photoresist layer on the silica substrate. Subsequent development of the photoresist, and reactive-ion etching into the silica substrate, formed the grating structure shown in the main scanning electron microscope (SEM) image in Fig. 2. The grating had a period of $398 \mathrm{~nm} \pm 5 \mathrm{~nm}$, and a depth of $134 \mathrm{~nm} \pm 9 \mathrm{~nm}$. The results show that the grating is strongly anharmonic, with a second-harmonic amplitude of approximately $20 \%$ of the fundamental, as can be seen in the inset of Fig. 2. The corrugations in the structure provided both distributed feedback and output coupling of the guided optical mode via second- and first-order Bragg scattering, respectively.

To complete the optical waveguide, a thin film of MEHPPV was spin coated from solution in chlorobenzene $(5 \mathrm{mg}$ MEH-PPV in $1 \mathrm{ml}$ chlorobenzene; spin speed of $1200 \mathrm{rpm}$ ) onto the silica. This formed a polymer layer with an average thickness of $\sim 100 \mathrm{~nm}$ as measured by a DekTak-3 surface profiler. As a result of the deposition process, the top surface 
(a) Air
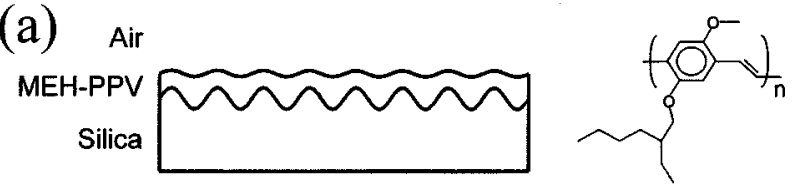

(b)

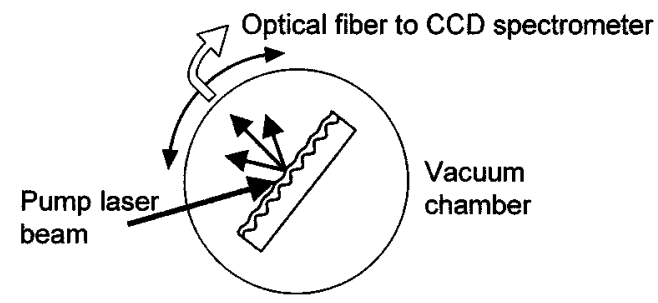

(c)

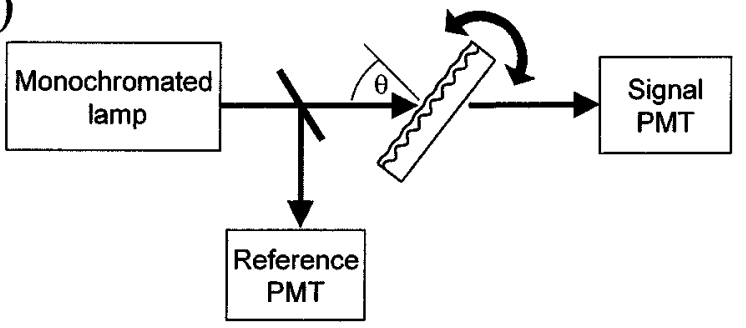

FIG. 1. (a) Schematic of laser structure and chemical structure of MEH-PPV; experimental configurations for (b) emission measurements, and (c) measurement of photonic band structure.

of the polymer layer was substantially more planar than the silica substrate, as shown in Fig. 2.

Immediately after fabrication, the sample was transferred to a vacuum chamber in which it was held under a dynamic vacuum of $\sim 10^{-4}$ mbar during the subsequent optical characterization. The experimental configuration for measurement of the emissive properties of the device is shown schematically in Fig. 1(b). The angular emission pattern from the structure was measured at excitation densities above, and well below, lasing threshold.

For the low power measurements, the sample was excited

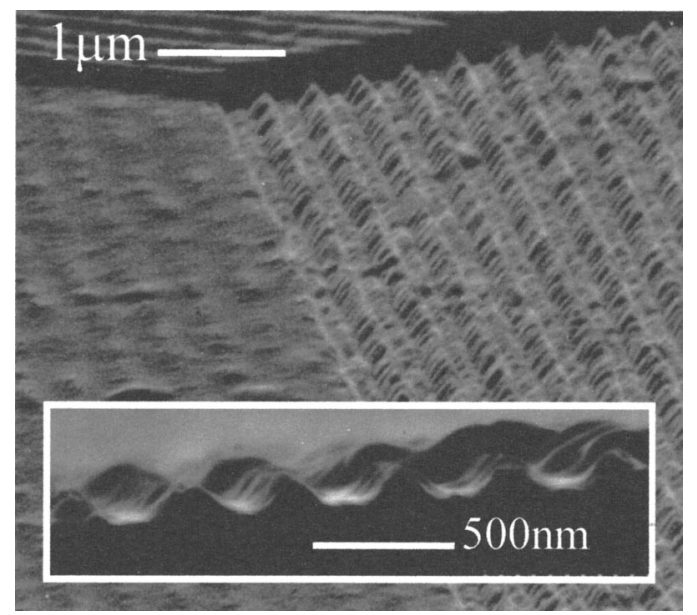

FIG. 2. SEM image of polymer laser, showing the bare silica substrate on the right and the top surface of the polymer film on the left. The inset shows an edge-on image of the cleaved grating structure. by the 488-nm line of an air-cooled argon-ion laser. The power of the pump laser was attenuated to less than $1 \mathrm{~mW}$, and the beam was focused to a circular spot of $\sim 100-\mu \mathrm{m}$ radius on the polymer surface. Photoluminescence spectra were detected using a fiber-coupled charge-coupled device (CCD) spectrometer for a range of polar angles to the normal to the waveguide plane. For these angular measurements, the 1-mm-diameter fiber was held at a distance of $50 \mathrm{~mm}$ from the excited region of the sample, thereby collecting an angular spread of the emission of $\sim 1^{\circ}$. The spectral resolution of the spectrometer was approximately $2.5 \mathrm{~nm}$.

For the high power measurements, the sample was excited at $500 \mathrm{~nm}$ by a dye laser that generated pulses of around $500-$ ps duration, at $10-\mathrm{Hz}$ repetition rate. The dye laser output was attenuated using neutral density filters to energies below $5 \mu \mathrm{J}$, and then focused onto the sample to a circular spot of $\sim 200 \mu \mathrm{m}$. The output from the laser was measured using the CCD spectrometer for a range of excitation powers. For these measurements the light was collected using a lens assembly connected to the end of the fiber in order to improve the signal to noise at the lowest average powers. The lens assembly collected an angular spread of the emission of $\pm 5^{\circ}$ centered on normal incidence. Other polarangular measurements of the polymer laser output (operating well above threshold) were collected using the bare end of the fiber, in a configuration identical to the photoluminescence measurements above.

The photonic band structure of the device was subsequently measured using the apparatus shown in Fig. 1(c) and described in detail in Ref. 16. The sample was mounted on a rotation stage and illuminated with the monochromated and collimated beam of a Xenon white light lamp. A polarizer placed between the lamp and the sample was used to discriminate between the excitation of TE and TM waveguide modes. The photonic mode structure of the sample could then be mapped out by measuring the absolute transmission as a function of angle and wavelength. Where the light couples into waveguide modes there is a reduction in transmission whose magnitude corresponds to the coupling strength. The transmission was measured by monitoring the intensity of the signal and reference beams with signal and reference photomultiplier tubes as the angle and wavelength were synchronously varied under computer control. The spectral resolution of the system was $\sim 1.5 \mathrm{~nm}$, limited by the spectral half width of the monochromator and the angular resolution achieved was $\sim 0.05^{\circ}$.

\section{RESULTS}

Photoluminescence spectra, taken at a low excitation power and a range of observation angles, are shown in Fig. 3 . The figure also shows the emission spectrum of a planar MEH-PPV film for comparison. It is clear that the presence of the corrugation drastically modifies the spontaneous emission, producing a pair of strong, narrow features that dominate the spectra. These narrow peaks move apart as the observation angle moves away from normal incidence, and are characteristic of emission that has been Bragg-scattered out of waveguide modes, ${ }^{17}$ in this case, the $\mathrm{TE}_{0}$ mode of the 


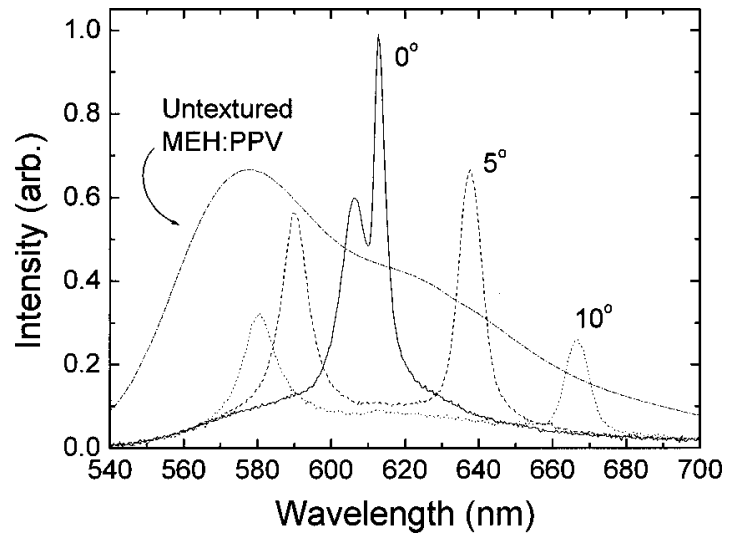

FIG. 3. Spectra of angle-dependent photoluminescence from the corrugated film, with a typical emission spectrum of an untextured MEH-PPV film for comparison (not to scale with the other spectra).

guide. A more detailed description of the coupling mechanism that gives rise to the angular dependence of the emission will be given in Sec. IV A.

A more complete set of data for the low power CW excitation at $488 \mathrm{~nm}$ is shown in Fig. 4 in a gray-scale plot of emission intensity as a function of wavelength and observation angle. This figure shows more clearly how the wavelengths of the narrow features change with angle, to map out two bands that anticross at an angle normal to the waveguide. The dip in the emission at this anticrossing (feature A in Fig. 4) is clearly visible in the normal incidence spectrum in Fig. 3. From Fig. 4 it is also apparent that, for emission close to the normal of the substrate, the scattered peaks in the long-wavelength band (feature B) are stronger and narrower than in the short-wavelength band (feature $\mathrm{C}$ ). This significance of this observation will become apparent when we compare it with data on the photonic band structure of the device and the lasing data in the discussion below, Sec. IV B.

Figures 5 and 6 shows the effect of excitation density on spectrum. Each spectrum was produced by integrating the

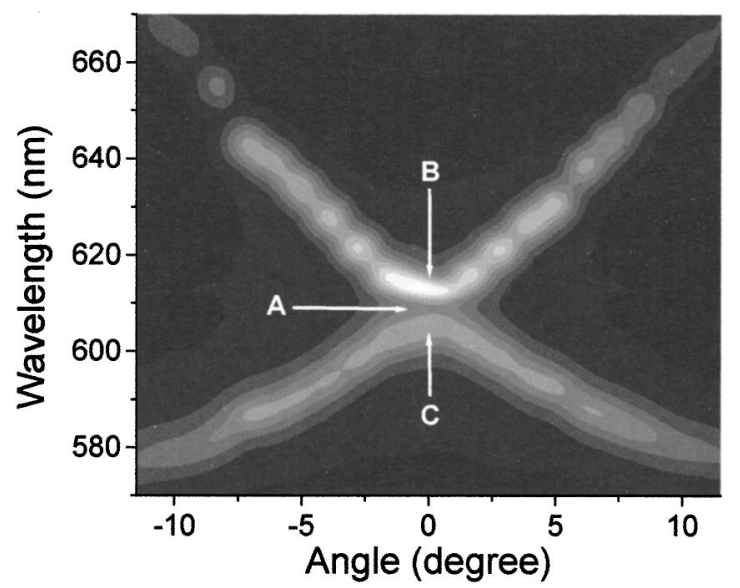

FIG. 4. Interpolated gray scale image of the angle-dependent photoluminescence; light regions represent the strongest emission. Labels A-C refer to (A) the dip in emission at the photonic band gap, (B) the long-wavelength emission band, and (C) the shortwavelength emission band.

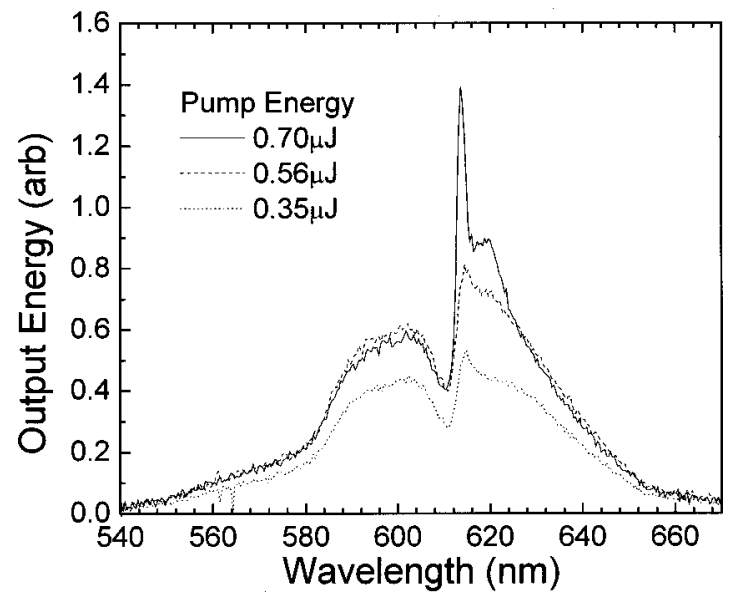

FIG. 5. Emission spectra for pump energies close to laser threshold.

emission over a small angular range $\left( \pm 5^{\circ}\right)$ about normal incidence. Figure 5 shows the transition in the spectrum as the emission from the device passes through the lasing threshold. The spectra show the clear dip in emission at 610 $\mathrm{nm}$ that was also evident in Fig. 3, and a small peak in the emission at the edge of the long-wavelength band. As the pump energy is increased up to $0.56 \mu \mathrm{J}$, the shape of the spectra remains largely constant. Above this level, however, a very narrow lasing peak begins to grow at $614 \mathrm{~nm}$ at the expense of increasing emission at other wavelengths, thus indicating the onset of band-edge laser operation. ${ }^{18}$

Figure 6 shows the effect of further increasing the pump energy, and the associated rise in the laser peak, as it becomes the dominant feature in the spectrum. A second, broader peak at $620 \mathrm{~nm}$ also appears above lasing threshold, arising from amplified spontaneous emission (ASE). The peak energy of the 614-nm emission is plotted as a function of pump energy in the inset of Fig. 6. There is a distinct kink in the curve where the device reaches threshold followed by a linear increase in output with pump energy; both features are characteristic of laser oscillation.

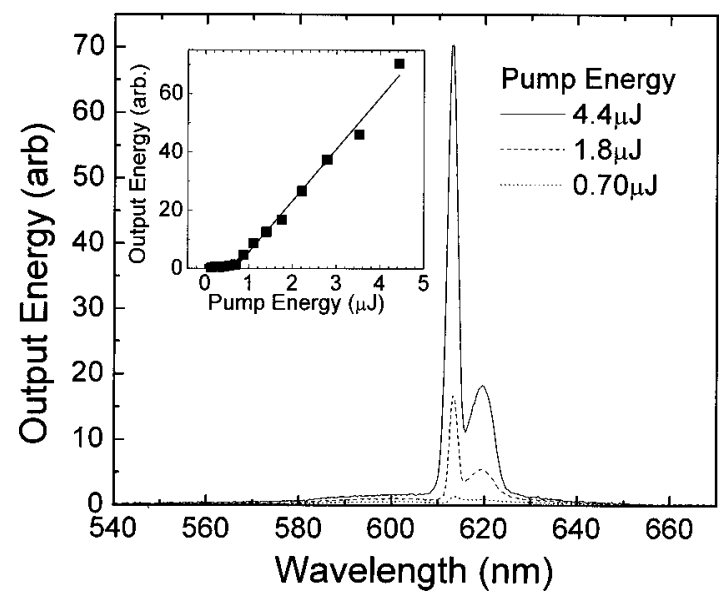

FIG. 6. Emission spectra for pump energies above laser threshold; inset shows the change in peak output intensity with pump energy. 


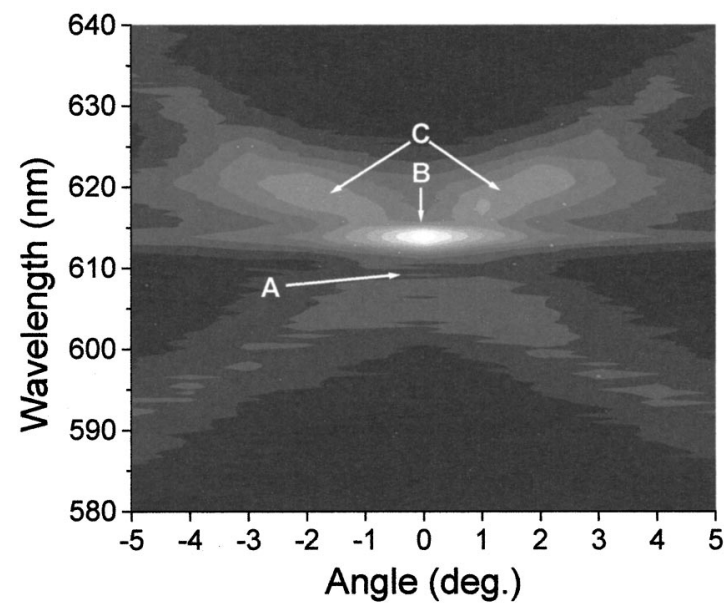

FIG. 7. Interpolated gray scale image of the angle-dependent emission when operating above laser threshold; light regions represent the strongest emission (logarithmic contour interval). Labels A-C refer to (A) the dip in emission at the photonic band gap, (B) the intense laser emission, and $(\mathrm{C})$ scattered ASE in the longwavelength emission band.

The angular dependence of the emission from the device when above laser threshold is plotted in Fig. 7 for a pump energy of $2.2 \mu \mathrm{J}$. As was previously seen in the low power excitation data, Fig. 4, the emission features two anticrossing bands with a dip in emission at $610 \mathrm{~nm}$ (feature A in Fig. 7). The laser mode is shown as an intense spot in the center of the figure (feature B), located at the edge of the longwavelength band. The angular spread of the laser beam in the $\phi=0^{\circ}$ plane (orthogonal to the grooves of the grating) is less than $1^{\circ}$, limited by the resolution of the detector. The scattered emission in the long-wavelength band (feature C) is substantially stronger than the short-wavelength band, due to scattering of the ASE feature (previously seen in Fig. 6) at a small angle to the normal.

The measured photonic band structure of the sample is shown in Fig. 8. This was recorded over a similar range to the photoluminescence to allow comparison with Figs. 4 and 7. The dark regions in Fig. 8 represent low transmission

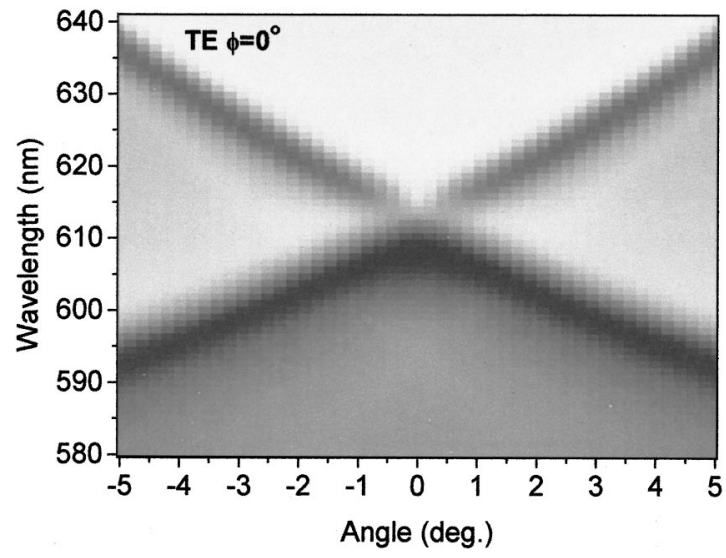

FIG. 8. Plot of the angle-dependent transmission of $s$-polarized light for $\phi=0$ (i.e., polarized parallel to the grating grooves); dark regions represent low transmission. through the sample due to the incident light scattering into waveguide modes, thus corresponding to coupling between the waveguide modes and free space radiation. The coupling occurs in two frequency bands that take a similar form to the emission profiles of Figs. 4 and 7. The strongest coupling occurs in the short-wavelength (high-frequency) band, which closely resembles the shape of the scattered photoluminescence. The long-wavelength band is much weaker, with a significant reduction in coupling strength close to normal incidence. We should emphasize that all the bands seen in the polymer emission and transmission measurements are the result of the scattered $\mathrm{TE}_{0}$ mode.

\section{DISCUSSION}

\section{A. Photonic band structure}

By comparing Figs. 4, 7, and 8, it is evident that the emission from the polymer laser is intimately linked with the photonic mode structure of the device. In order to explain the emission characteristics it is useful first to understand all the features in the photonic band dispersion.

The coupling of light between waveguide modes and free space radiation is due to Bragg scattering from the periodic grating structure. Specifically, two scattering processes are involved in the system: a first-order effect that couples light into (and out of) the waveguide, and a second-order effect that couples together counter-propagating waveguide modes. The fundamental Fourier component of the grating profile is responsible for the first process, while the second-harmonic spatial component makes a significant contribution to the second.

In Fig. 8, the wavelengths $\lambda$ for optimum coupling are found to vary strongly with the incidence angle. For angles $\theta$, well away from normal incidence, they follow the characteristic relation

$$
\frac{2 \pi}{\lambda} \sin (\theta)= \pm \frac{2 \pi n_{\mathrm{eff}}}{\lambda} \pm m \frac{2 \pi}{\Lambda} .
$$

In Eq. (1), $n_{\text {eff }}$ is the effective refractive index of the waveguide, $\Lambda$ is the fundamental grating period, and $m$ is an integer. Equation (1) is, in effect, a statement of momentum conservation in the plane of the waveguide; it includes multiples of the Bragg vector $k_{G}=2 \pi / \Lambda$ to account for the effect of the grating. The \pm signs in Eq. (1) allow for light coupling into both forward- and backward-propagating modes of the waveguide. For each incidence angle $\theta$, two distinct wavelengths may couple to the guide. The longer wavelength is scattered into a mode through an angle $(\theta$ $-\pi / 2$ ), while the shorter wavelength scatters through an angle $(\theta+\pi / 2)$ into the counter-propagating mode, leading to the two branches seen in Fig. 8.

For transmission close to normal incidence, second-order Bragg scattering also becomes important. The wavelengths that couple to the guide from normal incidence additionally experience Bragg scattering between counter-propagating modes. These guided modes interfere to set up a standingwave pattern with a periodicity of $\Lambda / 2$. From symmetry arguments, one finds that the structure supports two such 
standing waves. ${ }^{19}$ These have nodes either at the maxima or minima of the $\Lambda / 2$ harmonic component of the grating. Consequently, the standing waves have different energies, and hence different frequencies. A gap opens up in the band structure between these two band edges, within which guided modes always interfere destructively and so cannot propagate in the waveguide. This band-gap feature appears (as a reduction in coupling around $610 \mathrm{~nm}$ ) in the transmission plot in Fig. 8 because light can only couple into the waveguide where a mode exists.

The reduced coupling on the long-wavelength band edge arises from the spatial position of the standing wave field patterns relative to the fundamental component of the grating. ${ }^{19,20}$ The data exhibited in Fig. 8 is indicative of a symmetrical grating structure with the Fourier components of the cosine series that make up the corrugation being in phase. ${ }^{20}$

\section{B. Emission characteristics}

The measurements of the dispersion of the photonic bands provide significant insight to the interference mechanisms affecting transmission through the sample. Having now discussed the salient features of Fig. 8, we next use these phenomena to explain the emission spectra of the polymer film shown in Figs. 3-7. The angular dependence of the spontaneous emission in Fig. 4 shows a striking resemblance to the photonic band structure of Fig. 8. This implies that much of the light emitted from the front face of the polymer film arises from light that was first emitted into the $\mathrm{TE}_{0}$ waveguide mode and subsequently Bragg scattered to radiation by the grating. Importantly, the strength of the band edges as seen in the dispersion data, Fig. 8, and the emission data, Fig. 4, seem to be switched. To understand this it is perhaps easiest to look first in more detail at the lasing data.

Above threshold the angle dependent emission, Fig. 7, takes a general form similar to the photonic band diagram, Fig. 8. We note that the lasing emission occurs on the longwavelength band edge (see Figs. 5 and 6). This may seem somewhat surprising since the "strongest" band edge in the photonic band structure is the short-wavelength one. However, we recall that the short-wavelength band edge appears strongest in the data of Fig. 8 because it is better coupled to radiation than the long-wavelength band edge, as discussed above. This means that feedback will compete less favorably with coupling to radiation-i.e., the cavity losses for this band edge will be high. A direct consequence of the above is that the short-wavelength mode will have a higher threshold. This feature of second-order DFB lasers is well known in the field of inorganic lasers. ${ }^{20}$

As noted previously, Fig. 6 shows that there is a second peak in the emission spectrum when the device is above threshold, at $\sim 620 \mathrm{~nm}$ cf. the lasing peak at $614 \mathrm{~nm}$. We attribute this extra feature to amplified spontaneous emission, and note that it occurs at a wavelength corresponding to the shoulder in the untextured MEH-PPV emission spectrum in Fig. 3. The substantial narrowing from the broad spontaneous emission spectrum of MEH-PPV is due to preferential amplification of the wavelengths that experience the highest net gain. While the spontaneous emission is strongest around $580 \mathrm{~nm}$, at this wavelength there is still significant groundstate absorption that reduces the net gain. Thus the amplification tends to occur on the second vibronic feature in the spontaneous emission spectrum. Spectrally narrowed linewidths of under $10 \mathrm{~nm}$ are common in planar polymer films, ${ }^{1}$ and we have previously shown that these features may be tuned through the emission spectrum by control of the polymer film thickness. ${ }^{6}$ In the present corrugated waveguide the ASE wavelengths are Bragg scattered out of the guide at angles around $2^{\circ}$ to normal incidence, giving features $\mathrm{C}$ in Fig. 7.

Last we examine the spontaneous emission of Figs. 3 and 4. Surprisingly, we again find that the longer wavelength Bragg scattered feature dominates the spectrum. This occurs despite its associated mode experiencing weaker coupling to free space. Closer examination of the data, obtained for normal emission, in Fig. 3 reveals that the long-wavelength peak is also narrower than the short-wavelength one. This indicates a higher finesse for the mode responsible for the emission, consistent with the weaker coupling of the longwavelength mode to radiation. We therefore conclude that the stronger spontaneous emission is an artifact of the resonant transmission of the waveguide modes through the periodic structure.

Single frequency lasing has previously been reported in a second-order DFB polymer laser. ${ }^{12}$ The authors of that work attributed the spectral properties to the effect of periodic gain modulation, ${ }^{21}$ rather than index modulation. They argued that as the thickness of the polymer layer varied substantially, the gain in the waveguide was concentrated mainly in the thicker regions, in which most of the pump light was absorbed. They reported that the laser mode appeared very close to the center of the band gap, rather than on the band edge as found in the present work.

While periodic gain modulation may be present in our polymer laser, the evidence presented here strongly points to a system dominated by index modulation from the permanent microstructure. The laser mode was found to oscillate on the band edge and not in the gap (which would be characteristic of a complex-coupled system with significant gain modulation). Indeed, the photonic band structure was measured with low intensity light and therefore did not feature gain modulation, yet it can account for all of the spectral features in the emission. We should finally note that in the present system lasing occurs at the band edge closest to the peak optical gain (around $620 \mathrm{~nm}$ ), a factor that may also contribute to the frequency selection. However, in previous studies of similar laser structures, ${ }^{4}$ we found that the laser oscillated on the long-wavelength band edge, irrespective of the relative wavelengths of the stop band and peak gain. We therefore conclude that the difference in index coupling at the two band edges is the dominant frequency selection mechanism.

\section{CONCLUSIONS}

We have measured the emission characteristics and photonic band structure of a DFB laser, made from the conju- 
gated polymer MEH-PPV. We find that knowledge of the photonic dispersion curve explains how the substrate microstructure modifies both spontaneous and stimulated emission. The wavelength-scale corrugations provided both a distributed feedback in the plane of the film, and coupling between the waveguide mode and free space radiation. The structure showed a one-dimensional photonic band gap around 610 $\mathrm{nm}$, and at higher excitation powers DFB lasing occurred at one of the two band edges. The frequency selection is attributed to a difference in the output coupling of modes at the two edges of the band gap, a feature clearly illustrated in the photonic band structure data.

We have shown how direct measurements of the photonic

*Corresponding authors. Email address: idws@st-andrews.ac.uk and w.1.barnes@ex.ac.uk

${ }^{1}$ F. Hide, M. A. Diaz-Garcia, B. J. Schwartz, M. R. Andersson, Q. Pei, and A. J. Heeger, Science 273, 1833 (1996).

${ }^{2}$ N. Tessler, Adv. Mater. 11, 363 (1999).

${ }^{3}$ M. D. McGehee and A. J. Heeger, Adv. Mater. 12, 1655 (2000).

${ }^{4}$ G. A. Turnbull, T. F. Krauss, W. L. Barnes, and I. D. W. Samuel, Synth. Met. 121, 1759 (2001).

${ }^{5}$ B. J. Schwartz, F. Hide, M. R. Andersson, and A. J. Heeger, Chem. Phys. Lett. 265, 327 (1997).

${ }^{6}$ A. K. Sheridan, G. A. Turnbull, A. N. Safonov, and I. D. W. Samuel, Phys. Rev. B 62, R11 929 (2000).

${ }^{7}$ N. Tessler, G. J. Denton, and R. H. Friend, Nature (London) 382, 695 (1996).

${ }^{8}$ S. V. Frolov, M. Shkunov, Z. V. Vardeny, and K. Yoshino, Phys. Rev. B 56, R4363 (1997).

${ }^{9}$ M. D. McGehee, M. A. Diaz-Garcia, F. Hide, R. Gupta, E. K. Miller, D. Moses, and A. J. Heeger, Appl. Phys. Lett. 72, 1536 (1998).

${ }^{10}$ C. Kallinger, M. Hilmer, A. Haugeneder, M. Perner, W. Spirkl, U. Lemmer, J. Feldmann, U. Scherf, K. Mullen, A. Gombert, and V. Wittwer, Adv. Mater. 10, 920 (1998). band structure can be used to explain the spectral features of spontaneous and stimulated emission from a microstructured polymer waveguide. The type of measurements presented here can be a powerful tool for understanding the emission from polymer microstructures, and should aid the design of low threshold organic lasers suitable for electrical excitation.

\section{ACKNOWLEDGMENTS}

We are grateful to EPSRC (Grants GR/M73903/01 and GR/M73880/02), the Royal Society, and the Nichol Trust for financial support. The authors wish to thank Ian Hooper for assistance with the reactive ion etching.

${ }^{11}$ S. Riechel, C. Kallinger, U. Lemmer, J. Feldmann, K. Gombert, V. Wittwer, and U. Scherf, Appl. Phys. Lett. 77, 2310 (2000).

${ }^{12}$ M. Meier, A. Mekis, A. Dodabalapur, A. Timko, R. E. Slusher, J. D. Joannopoulos, and O. Nalamasu, Appl. Phys. Lett. 74, 7 (1999).

${ }^{13}$ F. Hide, B. J. Schwartz, M. A. Diaz-Garcia, and A. J. Heeger, Chem. Phys. Lett. 256, 424 (1996).

${ }^{14}$ S. V. Frolov and Z. V. Vardeny, Synth. Met. 111, 507 (2000).

${ }^{15}$ J. H. Schon, Ch. Kloc, A. Dodabalapur, and B. Batlogg, Science 289, 599 (2000).

${ }^{16}$ M. G. Salt and W. L. Barnes, Opt. Commun. 166, 151 (1999).

${ }^{17}$ A. N. Safonov, M. Jory, B. J. Matterson, J. M. Lupton, M. G. Salt, J. A. E. Wasey, W. L. Barnes, and I. D. W. Samuel, Synth. Met. 116, 145 (2001).

${ }^{18}$ J. P. Dowling, M. Scalora, M. J. Bloemer, and C. M. Bowden, J. Appl. Phys. 75, 1896 (1994).

${ }^{19}$ W. L. Barnes, T. W. Priest, S. C. Kitson, and J. R. Sambles, Phys. Rev. B 54, 6227 (1996).

${ }^{20}$ R. F. Kazarinov and C. H. Henry, IEEE J. Quantum Electron. 21, 144 (1985).

${ }^{21}$ H. Kogelnik and C. V. Shank, J. Appl. Phys. 43, 2327 (1972). 